\title{
Structural and Optical properties of Multiwalled Carbon Nanotubes Modified by DBD Plasma at Atmospheric Pressure
}

\author{
Norain Sahari ${ }^{1}$, Zolkafle Buntat ${ }^{*^{2}}$, Zulkifli Azman ${ }^{3}$, Zainuddin Nawawi $^{4}$, \\ Muhammad Abu Bakar Sidik ${ }^{5}$, Muhammad Irfan Jambak ${ }^{6}$ \\ $1,2,3$ Institute of High Voltage and High Current, Faculty of Electrical Engineering, Universiti Teknologi \\ Malaysia, 81310 UTM Johor, Skudai, Johor, Malaysia, \\ ${ }^{1,2}$ Department of Power, Faculty of Electrical \& Electronic, Universiti Tun Hussein Onn, 86400, Parit Raja, \\ Johor, Malaysia \\ ${ }^{4.5,6}$ Department of Electrical Engineering, Faculty of Engineering, Universitas Sriwijaya, South Sumatera, \\ Indonesia. \\ ${ }^{*}$ Corresponding author, e-mail: zolkafle@utm.my
}

\begin{abstract}
Structure, chemical, and physical properties of Multiwalled Carbon Nanotubes (MWCNTs) after modification by dielectric barrier discharge (DBD) at atmospheric pressure is investigated using Transmission Electron Microscopy (TEM), Raman and Uv-vis-NIR spectroscopy. Effects of plasma treatment time on MWCNTs are analyzed. TEM result shows that during the short period of plasma treatment time of 5 minutes, the tube surface experienced a few damages. With increase in plasma treatment time, the tube surface is damaged to a certain extent. Intensity ratio, $I_{D} / I_{G}$ through Raman analysis shows a good agreement with TEM. The values of $I_{D} / I_{G}$ of the modified MWCNTs are larger than those of pristine MWCNTs. An increase of $I_{D} / I_{G}$ indicates that considerable defects are produced on the surfaces of MWCNTs. The treated MWCNTs has energy band gap compared to zero band gap of untreated MWCNTs. It is believed that the defect site of MWCNTs can modify the electronics properties of MWCNTs from being metallic to semiconducting structure, which is applicable for almost all electronics device applications.
\end{abstract}

Keywords: DBD plasma, atmospheric pressure, modified MWCNT, energy band gap

Copyright $\odot 2018$ Universitas Ahmad Dahlan. All rights reserved.

\section{Introduction}

Carbon nanotubes (CNTs), first discovered by lijima [1] in 1991, are one of the most potential candidates for many new devices such as sensors [2-3], field effect transistors (FET) [4-6] and field emission displays [7]. CNT can be divided into two types according to the number of their wall layer; single-walled carbon nanotubes (SWCNTs) and multi-walled carbon nanotubes (MWCNTs). SWCNTs are made up of one-atom thick layer of graphene which rolls up to create a seamless cylinder with diameter of $0.4-2 \mathrm{~nm}$ and length in order of 1 100 microns [8]. MWCNTs are composed of multiple layers of graphene wrapped up together to form a cylindrical shape that is concentrically nested. The structure of CNTs may have seamless one or more coaxial cylindrical sheets of graphene with an aspect ratio typically larger than 100. These crystalline, tubular carbon structures have amazing mechanical, chemical, thermal, and electrical properties [9], [10]. Since the finding of the carbon nanotubes in 1991 by lijima [1], researchers have been struggling for better comprehension of their basic properties and applications in numerous fields of science and technology [4], [11-12]. CNTs have excellent mechanical, electrical and thermal properties because of their strong bonding structure of carbon atoms. A carbon atom in a CNT possesses six electrons, two of which are located in the first orbital and the other four fill the $\mathrm{sp}^{2}$ orbital [13-14]. Still, there are many major obstacles in exploring the possibility of CNTs in industrial applications such as being uncontrollable in its chirality- a key factor whether the CNT will be metallic or semiconducting. Semiconducting CNT is important for almost all electronics device applications, such as in field effect transistor (FET) because only semiconducting channel conductance can be controlled or modulated by the gate [15]. 
Theoretically, SWCNT can be either metallic or semiconducting depending on its chirality. Its band gap also can vary from zero to about $2 \mathrm{eV}$. Band gap of CNTs are subject to circumferential quantum confinement, which depends on the diameter of the tube. MWCNT, which consists of multiple roll layers, is usually classified as zero band gap due to the restriction on the relative diameters of each individual tube. Initial study by Martel et al [16] reported that MWCNTs typically show no gate effect due to its large tube diameter, but structural defects of MWCNTs can make them operate as FET.

The analysis on defects and impurities into CNT could offer a possible route to change and tune its electronics properties. The creation of new energy levels in the band gap with associated electronics states is an important stepping-stone in the manufacturing of electronics devices. For instance, nitrogen doping of carbon nanotubes can be used to activate the v-CNT tips, which allows the tuning of its electronics properties [17]. Exposing nanotube films to vapors from harsh chemicals can modify the electronics properties of CNT [18]. The substitution of carbon atoms by boron dopants is capable to modify the electrical and structural properties of CNT [19]. Many methods have been developed to modify and create defects onto MWCNTs. Several methods have been used to modify the surface characteristics of CNTs such as electrochemical [20], fluorination [21], polymer wrapping [22] and plasma treatment [23-24]. Plasma treatment offers several benefits including short duration of treatment time, nonpolluting process, and ability to operate at room temperature. It's also offer a wide range of grafted functional group depending on the plasma parameters such as type of gases used, applied power, duration of treatment and gas pressure. During the treatment process, the $\mathrm{C}=\mathrm{C}$ bond of CNTs is attacked by the excited molecules and generates radicals during plasma discharge, producing open ends and defect sites as main sites for functionalization [23]. Among the various methods used to generate plasma, dielectric barrier discharge (DBD) plasma method is the simplest and most flexible plasma. This technique is characterized by the existence of at least one dielectric material in contact with the discharge between cylindrical or two planar electrodes connected to high voltage power supply.

In this study, defects structures of MWCNT were created using dielectric barrier discharge plasma treatment. The aim of the present work is to study the possibility of modifying the electronics properties of metallic MWCNT by using DBD treatment at atmospheric pressure. The surface chemical and physical properties changes occurring on MWCNT after modification were investigated by using TEM, Raman Spectroscopy and UV-vis-NIR Spectroscopy.

\section{Experimental Details}

Multi-walled carbon nanotubes (MWCNTs) were prepared by chemical vapour deposition with the Co-Mo/MgO as catalyst with diameter distribution of $12.8 \pm 4.2 \mathrm{~nm}$ [25]. The experimental apparatus for DBD treatment was specifically made as plate-to-plate type having an electrode size of $60 \mathrm{~mm}$ diameter with $5 \mathrm{~mm}$ thickness. Both electrodes were covered by wire mesh, specified as 325 meshes per inch and $0.035 \mathrm{~mm}$ wire diameter (\#325, 0.035). Wire mesh was used to cover the electrodes so that more homogeneous discharge entire the electrode could be obtained [26]. Glass plate with $2 \mathrm{~mm}$ thickness was used as dielectric barrier. The gap spacing and the helium flow rate were kept constant at $2 \mathrm{~mm}$ and $1 \mathrm{l} / \mathrm{m}$, respectively. Plasma was produced at a discharge voltage of $3-15 \mathrm{kV}$ and a frequency $50 \mathrm{~Hz}$ with a step-up transformer. The voltage applied to the electrodes was measured using a high voltage probe. The discharge current and lissajous figure were measured by placing a $50 \Omega$ resistor and 0.22 $\mu \mathrm{F}$ capacitor between the bottom electrode and ground, respectively. The waveform of the applied voltage and discharge current were recorded using a PicoScope 5242A. The MWCNTs samples were exposed to the DBD plasma for various time intervals in the range of 5-10 minutes. In Figure 1(a), the schematic of the experimental setup is presented. Meanwhile, Figure 1(b) shows the applied voltage and current characteristics of DBD at $2 \mathrm{~mm}$ discharge gap and $6 \mathrm{kV}$ of peak voltage, and the image of generated plasma. From the waveform, the mode of the discharge is in filamentary mode due to the existance of several pulse in both positive and negative half cycles [26]. 


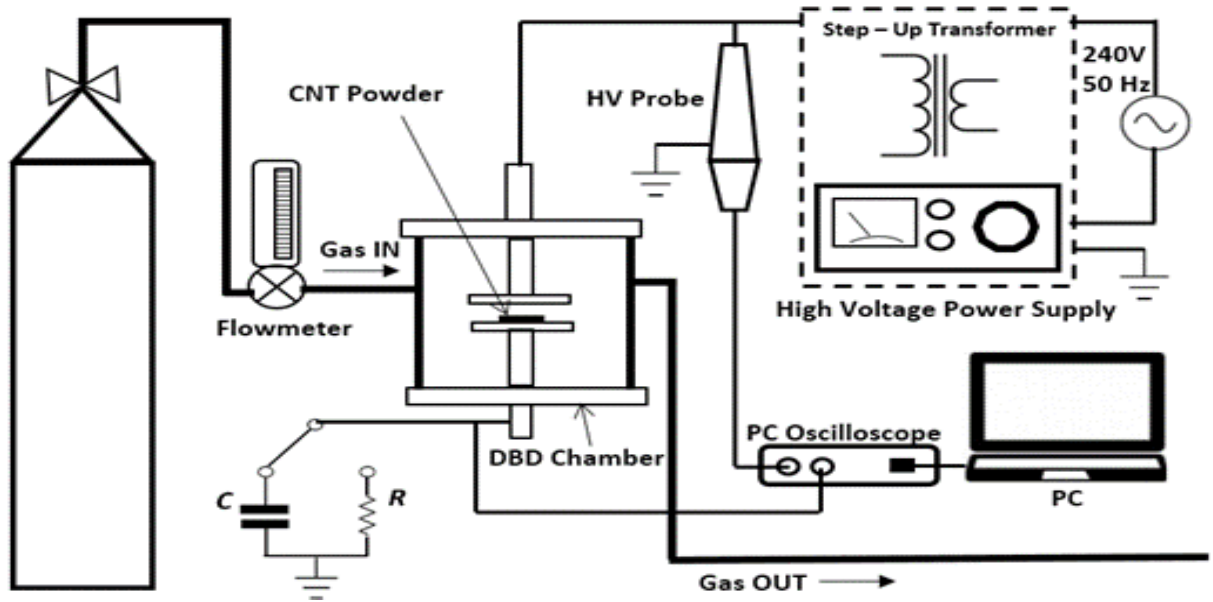

(a)
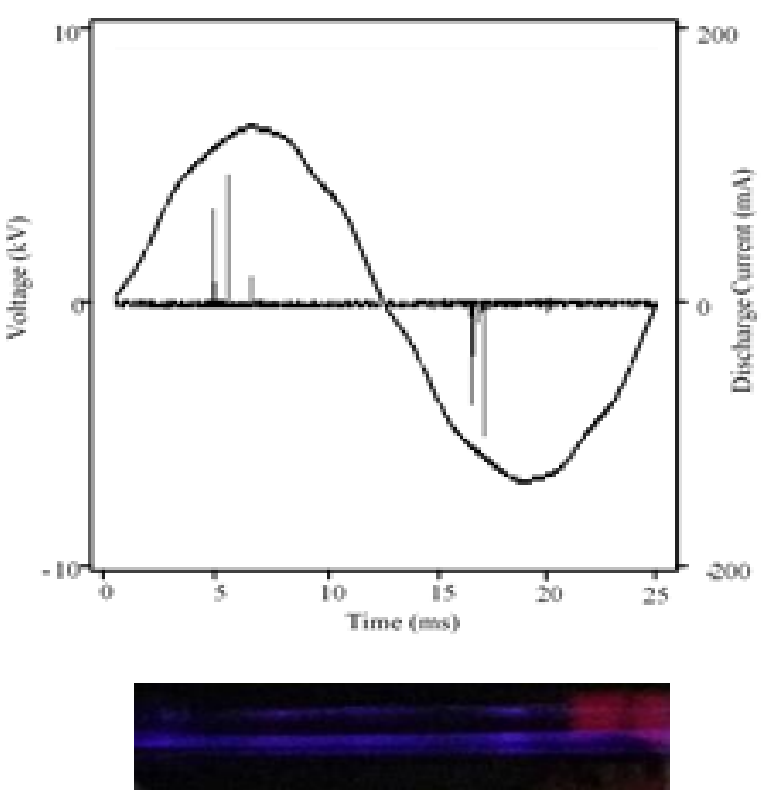

(b)

Figure 1. (a) Schematic of the experimental set-up for DBD plasma treatment; (b) Voltage and discharge current waveform of DBD, and the image of generated plasma

The structures of pristine and modified MWCNTs were characterized by using Transmission Electron Microscopy (TEM) and Raman spectroscopy. UV-vis-NIR Spectroscopy studies were employed to investigate the energy band gap of modified MWCNTs using Tauc's plot. TEM was performed by dipping a holey-carbon TEM grid into a colloidal suspension of the MWCNTs. Sample images were shot using a TEM Hitachi HT7700 field-emission gun, transmission electron microscope operating at $297 \mathrm{kV}$. Using a CCD camera, which is mounted on a GIF 200 electron energy loss spectrometer, the images were captured.

Raman spectroscopy studies were conducted in order to investigate the defect structure of the sample. The MWCNT samples were analysed by using a Raman Horiba Xplora Plus system with excitation line set to $\lambda=532 \mathrm{~nm}$ and magnification set to 40x. Detailed scans from $300 \mathrm{~cm}^{-1}$ to $4000 \mathrm{~cm}^{-1}$ were conducted on each MWCNT sample. The ultraviolet-visible-near infrared (UV-vis-NIR) spectra were acquired using a Shimadzu UV Spectrophotometer with samples being prepared by tip sonication of the MWCNTs for 20 minutes. 


\section{Results \& Discussions}

Practically, charged ions and electrons produced during the plasma treatment should affect the CNT surface morphology physically and chemically. In this study, structural damage (e.g. sidewall damage) was induced by plasma treatment, and surface modifications were generated. In addition, the degree of damage was observed increased along with plasma processing time, obviously demonstrated from TEM measurements. As seen in Figure 2, there was an increase in defects on the MWCNTs surface in 5 minuntes and 10 minutes treatment time respectively, compared to smooth surface of pristine MWCNTs. Through the short 5 minutes duration of plasma treatment time, the tube surface experienced some damages and structural distortion as indicated by red arrow. With increase in plasma treatment time, the morphology of CNT evidently changed. When treated for 10 minutes, the tube surface got damaged to a certain extent. The number of structural distortion got increased and the structure of MWCNTs become disordered. During plasma treatment, defect structures were created on the MWCNT graphite layers due to the collision of high energetic ions from the plasma. Consequently, this led to other defects such as dangling bonds, and since these interlayers cross-link, they may experience sp3 defects, leading to bents or irregular graphitic structures. The interaction between excited species during plasma treatment such as radicals, electrons, ions and UV light with the CNT surface can break the C-C bonds and form active sites for the bonding of functional group.

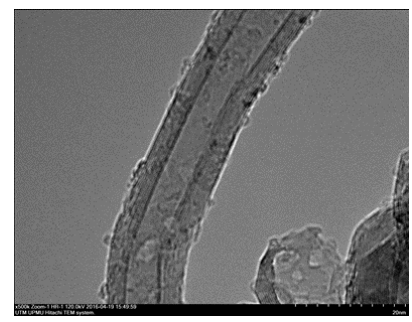

(a)

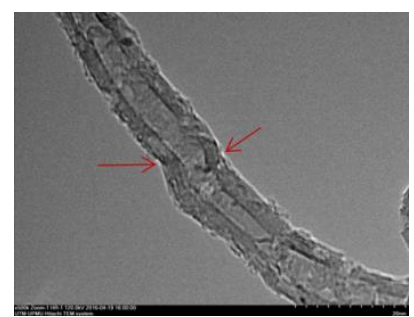

(b)

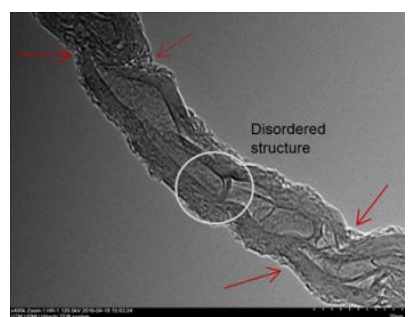

(c)

Figure 2. TEM micrographs of (a) pristine MWCNTs (b) after 5 minutes treatment (c) after 10 minutes treatment

To demonstrate the presence of defects in the modified MWCNTs, Raman characterization was performed. The Raman D band (Disorder) was found at $1350 \mathrm{~cm}^{-1}$. Figure 3 shows the amorphous carbon and structural defects. Meanwhile, the G band (Graphite) was located at $1570 \mathrm{~cm}^{-1}$, related to graphite structures and stemmed from tangential shearing mode of the carbon atoms [27-28]. In the spectra of this MWCNT, D' band appeared at 1610 $\mathrm{cm}^{-1}$ as a small shoulder of the $G$ band. Similar to $D$ band, the $D$ ' band also had been ascribed as the disorder features of graphitic sheets. The details of Raman analysis is summarized in Table 1. The values given in parentheses in Table 1 are the full width at half maximum (FWHM) of the $D$ band. The decrease in FWHM of the $D$ band is suggestive of the decreased $\mathrm{sp}^{2}$ character. This decreasing pattern also corresponded to a decrease in the diameter of MWCNT, clearly related with a higher structural disorder. The fraction of integrated intensities of the D and $G$ bands, $I_{D} / I_{G}$, can be used to evaluate the density of defects in the CNT structure. With greater value of the $I_{D} / I_{G}$ ratio, there will be higher defect density in CNT [29].

The values of $I_{D} / l_{G}$ for the modified MWCNTs were larger than for pristine MWCNTs. The $I_{D} / l_{G}$ ratio for pristine MWCNTs was close to 0.67 , which then increased to 0.79 and 0.98 after 5 minutes and 10 minutes treatments, respectively. An increase of $I_{D} / l_{G}$ showed that significant defects were produced on the surfaces of MWCNTs. Thus, plasma surface modification indeed raises defects in the CNT structure. As shown by TEM in Figure 2, a large density of defects in the most outermost layer including wall irregularities, twisting and collapse had been created after modification process. This shows that results obtained from TEM analysis agreed with the results obtained by Raman. 
In order to demonstrate the functional group that may attach to the sidewall of MWCNTs, the FTIR spectrum measurements were performed on the pristine MWCNTs and after 10 minutes treatment. As shown in Figure 4, the transmittance at $3430 \mathrm{~cm}-1$ in both spectra was identified to $\mathrm{O}-\mathrm{H}$ stretching vibration, which could be due to the water absorption from the surroundings on MWCNTs surfaces. In the spectrum of the MWCNTs-He, the peak observed at $1210 \mathrm{~cm}-1$ is attributed to $\mathrm{C}-\mathrm{O}$ stretching while the presence of $\mathrm{COOH}$ group was confirmed by the presence of small peak at $1738 \mathrm{~cm}-1$ which is corresponding to $\mathrm{C}=\mathrm{O}$ bond.

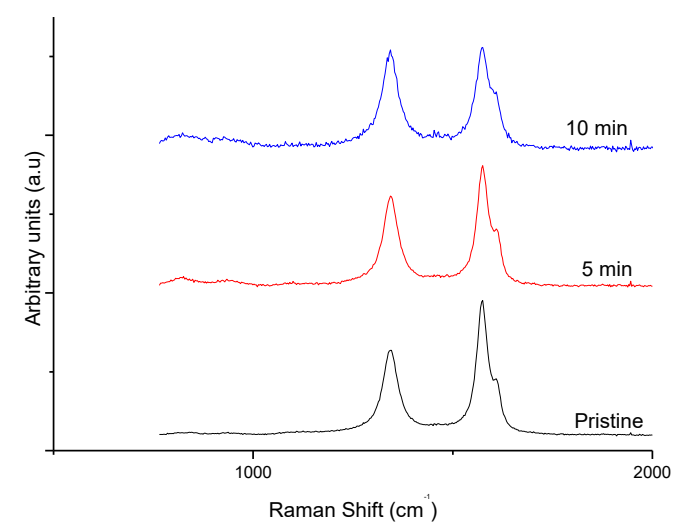

Figure 3. Raman Spectra of (a) Pristine MWCNTs (b) 5 minutes treatment (c) 10 minutes treatment

Table 1. Summary of Raman Analysis for Pristine and Modified MWCNT using DBD Plasma Treatment

\begin{tabular}{ccccc}
\hline Sample & $D$ band $\left(\mathrm{cm}^{-1}\right)$ & $\mathrm{G}$ band $\left(\mathrm{cm}^{-1}\right)$ & $D^{\prime}$ band $\left(\mathrm{cm}^{-1}\right)$ & $I_{D} / I_{G}$ \\
\hline Pristine & $1344(64.5)$ & 1575 & 1609 & 0.67 \\
5 minutes & $1344(56.0)$ & 1575 & 1609 & 0.79 \\
$\begin{array}{c}\text { treatment } \\
10 \text { minutes } \\
\text { treatment }\end{array}$ & $1344(44.9)$ & 1575 & 1609 & 0.98 \\
\hline
\end{tabular}

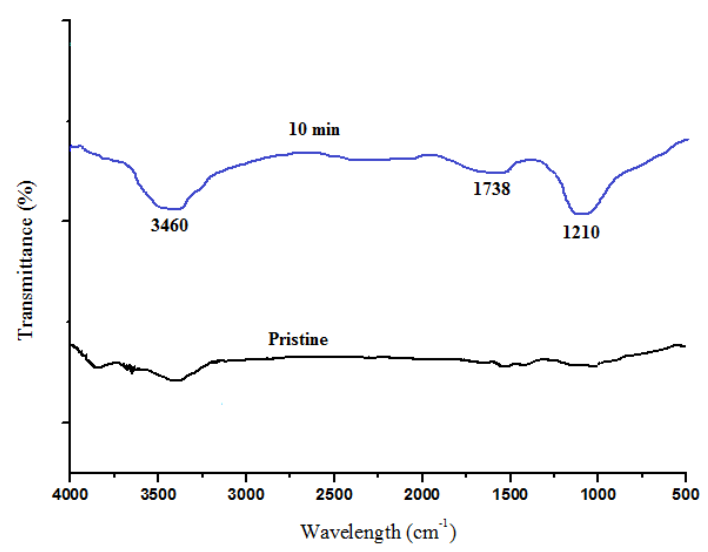

Figure 4. FTIR spectrum for pristine and after 10 minutes treatment

Generally, a pure He plasma does not comprise any chemically reactive species and not chemically terminated with carbon atoms during plasma treatment. In this study, plasma treatment was implemented at atmospheric pressure. Thus, all oxygen species incorporate to MWCNTs after plasma treatment can be attributed to the disruption of $\mathrm{O}_{2}$ and water vapor from 
ambient atmosphere. These results reveal that the impurities, oxygen and water vapor from surrounding of atmosphere contribute to the existence of defects in MWCNTs. Longer treatment will lead to higher defect structure and structural disordered.

Ultraviolet spectrum in Figure 5 shows the optical transmittance curves as a function of wavelength for MWCNT of different treatment times. The pristine MWCNTs demonstrated low transmittance intensity compared to treated MWCNTs. For 5 minutes and 10 minutes treatments of MWCNT, it was observed that high transmission $(T>40 \%)$ occurred in the wavelength range of 400-1000 nm, indicating the transmission of light through the MWCNTs intensification with reduction of energy. There was severe drop in transmittance at $300 \mathrm{~nm}$ corresponded to the $\pi$ plasmon absorbance.

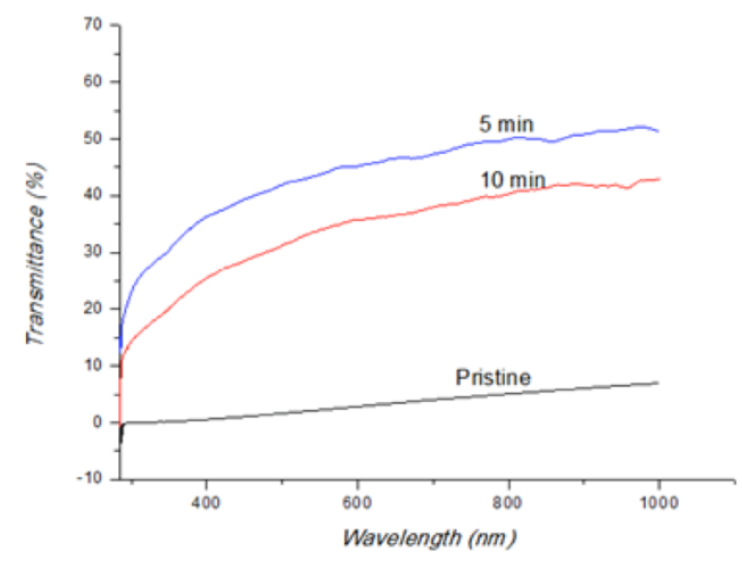

Figure 5. UV/vis/NIR Transmittance spectra for pristine MWCNTs, 5 minutes treatment and 10 minutes treatment

From UV-vis transmittance spectra, the band gap energy can be determined by using the Tauc relation. It is a convenient way of studying the optical absorption spectrum of a material. According to the Tauc relation, the absorption coefficient $(\alpha)$ for direct band gap material is given by:

$$
\alpha h v=B\left(h v-E_{g}\right)^{p}
$$

where $E_{g}$ is the band gap, constant $\mathrm{B}$ is difference for different transition, $h v$ is energy of photon and $p$ is an index that depends on the nature of electronics transition responsible for the reflection. The absorption coefficient ( $\alpha$ ) is defined by using the Lambert-Beer law. It can also be determined from the optical transmittance. The fundamental absorption, which relates to the transition of electrons from valence band to the conduction band, can be used to determine the energy band gap of material.

$$
\alpha=-\ln A / d
$$

where $A$ is the absorbance, $A=-\log T ; T$ is the transmittance determined from the UV-visible spectrum and $d$ is the path length of the quartz cuvette. The value of band gap was obtained from the extrapolation of the best linear parts of the curves at $\alpha=0$ near the band edge region.

Band gap is the most important parameter in the physics of semiconductors, especially for those widely used in nanoelectronics applications [1-3]. For individual CNT, theoretical calculations and experimental results have shown that the fundamental energy gap is zero in metallic CNT, and on the order of about $0.5 \mathrm{eV}$ in semiconducting CNT [4-6]. All the bands have a direct band gap, which permits electronic transitions between the valence and conduction band to proceed without the interference of photons. From Tauc plot in Figure 6, it can be seen that the pristine MWCNT has zero band gap, which denotes that the MWCNTs are metallic. While for 5 minutes plasma treatments the band gap increases to about $0.14 \mathrm{eV}$. The band gap decrease to $0.08 \mathrm{eV}$ 
for MWCNTs treated at 10 minutes duration. This transition of band gap to the value larger than zero shows that plasma surface treatment is potentially used as method to modify the electronics structure of MWCNTs from being metallic to semiconducting structure. The defects site of MWCNTs will modify the electronics states and optical transitions of the CNTs, which will result in forbidden transitions rather than allowed transitions of metallic CNTs. Nevertheless, it is worth to note that it is sufficient to have only little structural defects to modify the electronic properties of MWCNTs. Therefore, the duration for plasma treatment supposedly to be monitored and controlled so that higher structural defect can be avoided that seems to revert the MWCNTs from semiconducting back to the metallic CNTs. Based on the above finding, the recommended time duration of plasma treatment should be less than 5 minutes in order to obtain the fundamental energy gap of $0.5 \mathrm{eV}$ for semiconducting CNT.

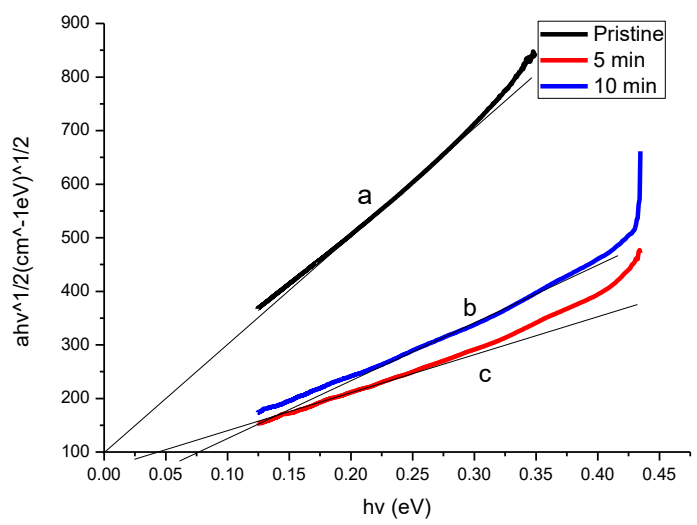

Figure 6. Tauc plot $a h v^{1 / 2}$ as a function of $h v$ for (a) pristine (b) 5 minutes treatment (c) 10 minutes treatment

\section{Conclusion}

MWCNTs have been successfully modified via a relatively simple technique. TEM, Raman and FTIR observations confirm that structural defects will be created after modification process using DBD at atmospheric pressure. There will cause a higher degree of disorder in the treated MWCNTs structure, as indicated by the Raman spectra. The treated MWCNTs also have energy band gap compared to zero band gap of untreated MWCNTs. It is believed that the defect site of MWCNTs can modify the electronics properties of MWCNTs. This study also suggest that longer time treatment in atmospheric pressure should be avoided in order to prevent severe structural damage of MWCNTs.

\section{Acknowledgments}

The authors gratefully acknowledge the financial support from Ministry of Education Malaysia and Universiti Teknologi Malaysia (Grant No R.J130000.7809.4F382) and Universitas Sriwijaya Collaboration Grant 2016 (R.J130000.7323.4B276)

\section{References}

[1] S lijima. Helical microtubules of graphitic carbon. Nature. 1991; 354: 56-58.

[2] YJ Kwon, HG Na, SY Kang, SW Choi, SS Kim, HW Kim. Selective detection of low concentration toluene gas using Pt-decorated carbon nanotubes sensors. Sensors and Actuators B: Chemical. 2016; 227(5): 157-168.

[3] A Abdelhalim, M Winkler, F Loghin, C Zeiser, P Lugli, A Abdellah. Highly sensitive and selective carbon nanotube-based gas sensor arrays functionalized with different metallic nanoparticles. Sensors and Actuators B: Chemical. 2015; 220: 1288-1296.

[4] P Bondavalli, P Legagneux, D Pribat. Carbon nanotubes based transistors as gas sensors: state of the art and critical review. Sensors and Actuators B: Chemical. 2009; 140: 304-318. 
[5] M Claus, D Gross, M Haferlach, M Schröter. Critical review of CNTFET compact models. NSTINanotech (Workshop on Compact modeling), 2012: 770-775.

[6] B Sankara Rao, CS Sunandana, VVSS Srikanth, TN Rao, PK Jain, K Bikshalu, et al. Highperformance Carbon Nanotube Field Effect Transistors with High k Dielectric Gate Material. Materials Today: Proceedings. 2015; 2: 4457-4462.

[7] JY Pan, YL Gao, CF Yang. Using different supporting mediums to improve the field emission characteristics of carbon nanotubes. Microelectronic Engineering. 2015; 148: 34-39.

[8] MS Dresselhaus, G Dresselhaus, PC Eklund, Science of fullerenes and carbon nanotubes: their properties and applications: Academic press, 1996.

[9] JP Salvetat, JM Bonard, N Thomson, A Kulik, L Forro, W Benoit, et al. Mechanical properties of carbon nanotubes. Applied Physics A. 1999; 69: 255-260.

[10] N Sinha and JTW Yeow. Carbon nanotubes for biomedical applications. NanoBioscience, IEEE Transactions on. 2005; 4: 180-195.

[11] Y Wang, JT Yeow. A review of carbon nanotubes-based gas sensors. Journal of Sensors. 2009.

[12] WB Choi, DS Chung, J HKang, HY Kim, YW Jin, IT Han, et al. Fully sealed, high-brightness carbonnanotube field-emission display. Applied Physics Letters. 1999; 75: 3129-3131.

[13] MS Dresselhaus, G Dresselhaus, P Eklund, A Rao, Carbon nanotubes: Springer, 2000.

[14] $\mathrm{RH}$ Baughman, AA Zakhidov, WA de Heer. Carbon nanotubes--the route toward applications. Science. 2002; 297: 787-792.

[15] J Charlier, P Eklund, J Zhu, A Ferrari. Carbon Nanotubes: Advanced Topics in the Synthesis, Structure, Properties and Applications. Jorio, A. 2008.

[16] Ra Martel, T Schmidt, H Shea, T Hertel, P Avouris. Single-and multi-wall carbon nanotube field-effect transistors. Applied Physics Letters. 1998; 73: 2447-2449.

[17] M Scardamaglia, C Struzzi, FJ Aparicio Rebollo, P De Marco, PR Mudimela, JF Colomer, et al. Tuning electronic properties of carbon nanotubes by nitrogen grafting: Chemistry and chemical stability. Carbon. 2015; 83(3): 118-127.

[18] D Janas, S Boncel, AA Marek, KK Koziol. A facile method to tune electronic properties of carbon nanotube films. Materials Letters. 2013; 106: 137-140.

[19] HZ Geng, KK Kim, C Song, NT Xuyen, SM Kim, KA Park, et al. Doping and de-doping of carbon nanotube transparent conducting films by dispersant and chemical treatment. Journal of Materials Chemistry. 2008; 18: 1261-1266.

[20] C González-Gaitán, R Ruiz-Rosas, E Morallón, D Cazorla-Amorós. Functionalization of carbon nanotubes using aminobenzene acids and electrochemical methods. Electroactivity for the oxygen reduction reaction. International Journal of Hydrogen Energy. 2015; 40: 11242-11253.

[21] S Mallakpour, A Zadehnazari. A facile, efficient, and rapid covalent functionalization of multi-walled carbon nanotubes with natural amino acids under microwave irradiation. Progress in Organic Coatings. 2014; 77: 679-684.

[22] SA Ntim, O Sae-Khow, FA Witzmann, S Mitra. Effects of polymer wrapping and covalent functionalization on the stability of MWCNT in aqueous dispersions. Journal of Colloid and Interface Science. 2011; 355: 383-388.

[23] Z Rastian, AA Khodadadi, F Vahabzadeh, C Bortolini, M Dong, Y Mortazavi, et al. Facile surface functionalization of multiwalled carbon nanotubes by soft dielectric barrier discharge plasma: Generate compatible interface for lipase immobilization. Biochemical Engineering Journal. 2014; 90: 16-26.

[24] LG Nair, AS Mahapatra, N Gomathi, K Joseph, S Neogi, CPR Nair. Radio frequency plasma mediated dry functionalization of multiwall carbon nanotube. Applied Surface Science. 2015; 340: 6471.

[25] WM Yeoh, KT Lee, AR Mohamed, SP Chai. Production Of Carbon Nanotubes From Chemical Vapor Deposition Of Methane In A Continuous Rotary Reactor System. Chemical Engineering Communications. 2012; 199: 600-607.

[26] S Okazaki, M Kogoma, M Uehara, Y Kimura. Appearance of stable glow discharge in air, argon, oxygen and nitrogen at atmospheric pressure using a $50 \mathrm{~Hz}$ source. Journal of Physics D: Applied Physics. 1993; 26: 889.

[27] AM Rao, A Jorio, MA Pimenta, MSS Dantas, R Saito, G Dresselhaus, et al.. Polarized Raman Study of Aligned Multiwalled Carbon Nanotubes. Physical Review Letters. 2000; 84: 1820-1823.

[28] A Jorio, G Dresselhaus, MS Dresselhaus, M Souza, MSS Dantas, MA Pimenta, et al.. Polarized Raman Study of Single-Wall Semiconducting Carbon Nanotubes. Physical Review Letters. 2000; 85: 2617-2620.

[29] S Costa, B Scheibe, M Rummeli, E Borowiak-Palen. Raman spectroscopy study on concentrated acid treated carbon nanotubes. Physica status solidi (b). 2009; 246: 2717-2720. 